\section{Valence and Reactivity}

\section{from a Correspondent}

As international symposium on valence and reactivity was held in Oxford under the auspices of the Chemical Society on January 9-1I. It was attended by nearly 200 members from more than 20 different countries.

Valence problems in the second half of the periodic table (Groups IV-VII) are bound up essentially with the role of $d$ electrons. As early as 1931, Pauling had proposed $s p^{3} d^{2}$ hybridization to account for the octahedral shapc of $\mathrm{SF}_{6}$. Further, the existence of complex ions such as $\mathrm{ClO}_{4}^{-}$and the non-existence of $\mathrm{FO}_{4}^{-}$are argued as evidence that the lower-lying $3 d$ orbitals of $\mathrm{Cl}$ can be used more readily than the less accessiblo $3 d$ orbitals of F. Similar situations occur when reactivily is considered. 'Thus, as Professor R. F. Hudson of the University of Kent showed, some substitution reactions can occur around a silicon atom by a form of ring-closure which does not occur in corresponding carbon compounds; an explanation is possible in terms of $d$-orbital participation in $\mathrm{Si}$.

But the really important question is: are we forced to invoke $d$ orbitals in these situations, or are there alternative explanations? A careful study of several types of microwave experiments by Dr E. A. C. Lucken of Geneva showed that in many cases alternative explanations were possible.

Some situations, however, did seem to be fairly certain. Thus Professor E. H. Wiebenga of Groningen showed that a complete account of the polyhalogen molecules and ions (such as $\mathrm{BrF}_{3}, \mathrm{I}_{3}{ }^{-}$) can be provided without any use of $d$ orbitals; all that is needed is the concept of three-centre bonds and $p$ electrons. This definite situation has to be balanced by the equally convincing arguments of Professor N. L. Paddock of British Columbia, whose account of the phosphonitrilis ring systems such as the chlorides $\left(\mathrm{PCl}_{2} \mathrm{~N}\right)_{n}$ invokes $d$ orbitals in no fewer than two planes.

A reconciliation of these views is possible, as was shown by Professor C. A. Coulson of Oxford, if we say that $d \sigma$ bonding is seldom important in ground states of molecules, but $\mathrm{d} \pi$ is much more likely to be important. Nevertheless in certain excited states, as Professor H. H. Jaffé of Cincinnati pointed out, a study of ultraviolet spectra shows $d \sigma$ and $d \pi$ orbitals to be necessary.

But the size of these $d$ orbitals is critical. They must not be too large, as they are calculated to be for an isolated atom, or their overlap will be small and their contribution to the bonding negligible. Professors D. W. J. Cruickshank of Manchester and C. Zauli of Bologna have calculated that both the presence of ligand groups around a central atom, and the mutual spin coupling arrangements of the valence electrons, can provide a mechanism for contracting the size of a $d$ orbital. So also can the presence of a formal positive charge on the central nucleus. In this way there is a nice explanation for the greater likelihood of large valence numbers when highly electronegative ligands such as fluorine are used.

An important feature of this symposium was the all-round character of the discussion. Topics of this kind require a comprehensive view. In this case it was abundantly justified.

\section{Discussing Nucleotides}

\author{
from a Correspondent
}

ALтночян bad weather cut down the attendance at the inaugural meeting of the nucleotide discussion group of the Chemical Society held on January 9 in the Chemistry Department of the University of Birmingham, the programme was followed mainly as planned. Professor M. Stacey formally opened the meeting by welcoming the visitors and Dr A. S. Jones took the chair.

The first proceedings took the form of a business meeting to constitute formally the nucleotide group. Dr Jones reported that more than seventy people had expressed an interest in the formation of the group and that a provisional committee had met to make proposals about rules and the constitution. The object is "to encourage the discussion of the chemistry including the biolngical chemistry of nuclcosides, nucleotides and nucleic acids". The committee will consist of a chairman, secretary-treasurer and four members who would normally serve for three years. The following were elected: chairman, Dr A. S. Jones (Birmingham); secretary-treasurer, Dr D. W. Hutchinson (Warwick); committee members, Dr M. G. Blackburn (Sheffield), Dr D. M. Brown (Cambridge), Professor K. Burton (Newcastle) and Dr R. T. Walker (Birmingham). The committee is to arrange a meeting at some convenient time during the carly summer.

The inaugural lecture at Birmingham was delivered by Professor Lord Todd, who drew attention to the large amount of work carried out on the synthesis of nucleotide analogues and suggested reasons for their relatively poor chemotherapeutic properties. He indicated that more information is needed about the role of rare bases in nucleic acids and that better methods of forming phospho-diester linkages in oligonucleotides are also required. Among the methods used to determine nucleotide sequence, he suggested that procedurcs by which one base might be rendered visible in electron micrographs by the use of a specific chemical marker would be worth developing. On the biochemical side he suggested that further work is necessary in order to establish more about the nature of the infectivity of viruses: in particular to decide why some RNAs are infective while others are not.

There were also four contributed papers. Professor K. Burton (Newcastle upon Tyne) gave some results on the effect of specific chemical reagents on the acceptor activity of transfer RNA and pointed to the need for a mothod of determining the tertiary structure of transfer RNA. Dr T. L. V. Ulbricht (Twyford Laboratories) discussed the use of optical rotatory dispersion in the determination of the conformation of nucleosides and its application in the determination of the conformation of nucleic acids. Dr G. Shaw (Bradford) discussed investigations of the chemical reactions involved in the biosynthesis of purine nucleosides, in particular the carboxylation and decarboxylation of some substituted imidazoles. Dr A. S. Jones (Birmingham) described the synthesis of polymerie analogues of polynucleotides, including polyacrylate derivatives with purine and pyrimidine side chains, and polycarboxymethylthymidine. 'These polymers interact in solution with polynucleotides. 